\title{
Differences in Patient Characteristics among Men Choosing Open or Robot-Assisted Radical Prostatectomy in Contemporary Practice - Analysis of Surveillance, Epidemiology, and End Results Database
}

\author{
Jonas Schiffmann ${ }^{a, d}$ Alessandro Larcher ${ }^{a, e, f}$ Maxine Sun ${ }^{a}$ Zhe Tian $^{a}$ \\ Jérémie Berdugo ${ }^{a, b}$ Ion Leva ${ }^{a, b}$ Hugues Widmer ${ }^{c}$ Jean-Baptiste Lattouf ${ }^{c}$ \\ Kevin Zorn ${ }^{c}$ Alexander Haese ${ }^{d}$ Shahrokh F. Shariat ${ }^{g}$ Fred Saad $^{c}$ \\ Francesco Montorsi $^{\text {eff }}$ Markus Graefen ${ }^{d}$ Pierre I. Karakiewicz ${ }^{\text {a,c }}$ \\ ${ }^{a}$ Cancer Prognostics and Health Outcomes Unit, ${ }^{b}$ Department of Pathology, and ${ }^{c}$ Department of Urology, \\ University of Montreal Health Center, Montreal, Canada; ${ }^{\mathrm{d}}$ Martini-Clinic, Prostate Cancer Center Hamburg- \\ Eppendorf, Hamburg, Germany; ${ }^{~}$ Urological Research Institute, IRCCS Ospedale San Raffaele, and ${ }^{f}$ Department of \\ Urology, Vita-Salute San Raffaele University, Milan, Italy; ${ }^{9}$ Department of Urology, Medical University of Vienna, \\ Vienna, Austria
}

\section{Key Words}

Characteristics · Open radical prostatectomy · Pelvic lymph node dissection P Prostate cancer $\cdot$ Robot-assisted radical prostatectomy

\footnotetext{
Abstract

Objective: To examine characteristics of robot-assisted (RARP) and open radical prostatectomy (ORP) patients. Patients and Methods: We relied on the Surveillance, Epidemiology, and End Results-Medicare-linked database and focused on prostate cancer patients between 2008 and 2009. In multivariable logistic regression analyses, we predicted RARP. Results: Of 5,915 patients, 3,476 (58.8\%) underwent RARP and 2,439 (41.2\%) ORP. Patients within intermediate (OR 1.4, $p=0.01$ ) or highest (OR 1.5, $p=0.02$ ) education stra-
}

ta and those treated by surgeons with a high volume (OR 2.2, $p<0.001)$ were more likely to undergo RARP. Conversely, those residing in rural areas (OR 0.7, $p=0.005$ ) and those with clinical stage T2 or higher (OR 0.7, $p=0.006$ ) were less likely to undergo RARP. Additionally, patients from the Southwest were less likely to undergo RARP (OR 0.4, p < 0.001 ), but those from the Northern Plains were more likely to undergo RARP (OR 1.4, $p=0.02$ ) than their counterparts from the East. Finally, RARP patients were neither younger nor healthier than ORP patients. Conclusions: Several patient characteristics such as education, region of residence and population density affect the likelihood of RARP vs. ORP treatment. Similarly, clinical stage and surgeon characteristics also affect the assignment to one or other treatment modality.

(c) 2016 S. Karger AG, Basel

\section{KARGER}

(c) 2016 S. Karger AG, Basel

E-Mail karger@karger.com

www.karger.com/uin
Jonas Schiffmann, MD

Martini-Clinic Prostate Cancer Center

University Medical Center Hamburg-Eppendorf

Martinistrasse 52, DE-20246 Hamburg (Germany)

E-Mail j.schiffmann@ klinikum-braunschweig.de 


\section{Introduction}

Radical prostatectomy (RP) represents the standard of care for localized prostate cancer (PCa) $[1,2]$. Over the past decade, robot-assisted RP (RARP) has gradually replaced the traditional open RP (ORP) [3]. Despite the massive preference for RARP, there are no randomized data supporting the benefit of RARP relative to ORP. Available population data demonstrate short-term advantages of RARP relative to ORP, such as fewer blood transfusions, fewer complications and shorter length of hospital stay [4]. Additionally, RARP is at least equivalent [5] if not superior [6] to ORP in terms of margin rates [7]. Moreover, the advantage of pelvic lymph node dissection (PLND) extent and/or yield at RARP vs. ORP is also being debated $[8,9]$. For example, population-based studies observed an underuse of PLND at RARP [10, 11]. Conversely, institutional data support the comparable feasibility of PLND at RARP [12-14].

However, long-term data from the United States demonstrate urinary and erectile dysfunction benefits favoring ORP [15]. These observations question the strong preference toward RARP instead of ORP. Since treatment decisions might be made on a subjective basis, it may be difficult to determine the characteristics of patients who opt for RARP, instead of ORP [16]. Based on these considerations, we decided to examine patient, clinical, pathological and surgical characteristics of individuals who either opt for RARP or ORP, within the most recent Surveillance, Epidemiology, and End Results (SEER)-Medicare-linked database.

\section{Materials and Methods}

\section{Population Sources}

This study relied on the most recent version of the SEER-Medicare-linked database. The SEER registries cover approximately $28 \%$ of the United States population with Medicare administrative data. Medicare covers approximately $97 \%$ of Americans aged $\geq 65$ years. Linkage to the SEER database is complete for approximately $93 \%$ of cases. [17].

\section{Study Population}

Overall, 6,310 patients with histologically confirmed PCa (international classification of disease for oncology site code 61.9, histologic code 8140) aged 65 years or older treated with RP from October 1, 2008 to December 31, 2009 were identified from the SEER-Medicare database. This time frame was selected as a specific modifier code for robotic-assisted approach was introduced on October 1, 2008 (ICD-9 clinical modification procedure code: 17.42). The latter allows for accurate identification of RARP. Patients who underwent ORP were also abstracted. Exclusion criteria consisted of patients aged $>80$ years $(n=97)$, metastatic disease $(\mathrm{n}=26)$, unknown tumor stage $(\mathrm{n}=130)$, unknown tumor grade $(\mathrm{n}=123)$, and unknown PLND status $(\mathrm{n}=19)$. This resulted in 5,915 assessable patients.

\section{Covariates}

For each patient, age at diagnosis, race, population density (urban vs. rural), marital status (married vs. unmarried), 2000 census tract percent with 4 -year college education (very low: $\leq 14.3$, low: 14.4-25.4, high: $25.5-42.2$ and very high: $\geq 42.3 \%$ ), 2000 census tract annual median income, region of residence, pathological Gleason score, preoperative prostate-specific antigen (PSA), clinical and pathological tumor stage, PLND status, nodal stage, and surgical volume (low: $\leq 2$, intermediate: $3-6$ and high: $\geq 7$ cases/ year) were assigned using the SEER data. The Charlson comorbidity index (CCI) was derived from the Medicare claims 1 year prior to $\mathrm{PCa}$ diagnosis, and categorized as $0,1,2$, and $\geq 3$, using a previously validated algorithm [18].

\section{Statistical Analyses}

Means, medians and interquartile ranges were reported for continuous variables. Frequencies and proportions were reported for categorical variables. The independent $t$ test and chi-square tests were used to compare the statistical significance of differences in means and proportions, respectively.

Additionally, logistic regression analyses predicting RARP were performed. Adjustment was made for surgical volume, region of residence, education, population density, clinical tumor stage, age, PSA, CCI, race, marital status and income. All statistical tests were performed using $\mathrm{R}$. All tests were 2 -sided with a significance level set at $\mathrm{p}<0.05$.

\section{Results}

\section{Baseline Descriptives}

We identified 5,915 PCa patients treated with RP. Of these total number of patients, 2,439 (41.2\%) were treated with ORP and 3,476 (58.8\%) with RARP. Several important patient characteristics that distinguish between RARP and ORP were identified. RARP patients had a lower median age ( 68 vs. 69 years, $p=0.04$ ), without significant differences according to age categories $(p=0.2)$ compared to those who opted for ORP. RARP patients were more from the 2 highest income and education categories. Specifically, patients treated with RARP were more frequently from the annual income category ranging from (US\$) 50,955-69,389: RARP 26.0 vs. ORP $23.8 \%$ and that above 69,389: RARP 29.0 vs. ORP 19.7\% ( $<<0.001)$. Similarly, patients treated with RARP were more frequently from the second highest (RARP: 26.3 vs. ORP: $23.4 \%$ ) and highest education strata (RARP: 28.5 vs. ORP: $20.5 \%$ ) than their counterparts treated with ORP $(\mathrm{p}<0.001)$.

Additionally, patients treated with RARP more frequently resided in urban centers (90.6 vs. $84.2 \%$, p < 
0.001). Statistically significant differences emerged with respect to region of residence. For example, patients treated with RARP were less likely to reside in the Southwest of the United States compared to patients treated with ORP ( 3.4 vs. $11.5 \%, \mathrm{p}<0.001)$. Of note is the absence of statistically significant or clinically meaningful differences with respect to age $(65-66,67-68,69-71$ or $\geq 72$ years) and CCI $(0,1,2$ or $\geq 3)$.

Regarding the clinical characteristics of patients treated with RARP, we also identified several important observations. First, the serum PSA values of RARP patient were more frequently $\leq 10 \mathrm{ng} / \mathrm{ml}$ (RARP: 75.0 vs. $69.3 \%$, p < $0.001)$. Second, the clinical tumor stage distribution revealed that more RARP patients harbored stages cT2a or lower at diagnosis (RARP: 88.8 vs. ORP: 84.9\%, p < 0.001). Finally, RARP patients more frequently harbored a Gleason score $\leq 7$ ( 85.8 vs. $83.4 \%, \mathrm{p}<0.001)$.

Last but not least, we also identified important pathological and surgical differences between RARP and ORP patients. For example, RARP patients were more frequently diagnosed with pT3 stage than their ORP counterparts ( 24.4 vs. $21.8 \%, \mathrm{p}=0.006)$. The proportion of patients with missing pathological stage was lower at RARP (6.4 vs. 8.2\%). Similarly, the proportion of PLND at RARP was also statistically and significantly lower than at ORP (49.4 vs. $71.8 \%, \mathrm{p}<0.001$ ). As a consequence, the rate of pathologically proven lymph node invasion ( $\mathrm{pN} 1)$ at $\mathrm{RP}$ was significantly lower at RARP than at ORP (1.4 vs. $3.4 \%, \mathrm{p}<0.001)$. Finally, RARP patients were more frequently treated by urologists within the highest surgeon volume tertile than ORP patients $(42.5$ vs. $21.8 \%$, p < 0.001; table 1; fig. 1).

\section{Logistic Regression Analyses Predicting RARP}

In the second part of the analyses, we relied on multivariable logistic regression models to predict the odds of undergoing RARP instead of ORP. According to these analyses, 5 predictors achieved independent predictor status: education level, region of residence, urban vs. rural population density, clinical $\mathrm{T}$ stage and surgical volume.

Of patient characteristics (education, region of residence and population density), those within the highest and second highest education groups were respectively 1.5- and 1.4-fold more likely to undergo RARP than patients from the very low education group (all $\mathrm{p} \leq 0.02$ ). Region of residence, specifically patients from the Southwest of the United States had a lower probability of undergoing RARP relative to patients residing in the East (OR 0.4, 95\% CI 0.3-0.6, p < 0.001). Finally, patients residing in rural areas were $30 \%$ less likely to undergo an
RARP compared to their counterparts from urban centers (OR 0.7, 95\% CI 0.5-0.9, $\mathrm{p}=0.005$ ).

Of clinical variables, clinical tumor stage achieved independent predictor status. Specifically, patients with cT2c or higher were less likely to undergo an RARP then their counterparts with stage cT2a or lower (OR 0.7, 95\% CI $0.5-0.9, \mathrm{p}=0.006$ ). Additionally, individuals operated by surgeons within the highest surgical volume tertile were more likely to undergo RARP then their counterparts treated by surgeons in the lowest volume tertile (OR $2.2,95 \%$ CI 1.8-2.7, p < 0.001). Finally, neither age (65-66 vs. $\geq 72$ years: OR $0.8,95 \%$ CI $0.6-1.0, p=0.059)$ nor CCI (CCI 0 vs. $\geq 3$ : OR $0.9,95 \%$ CI $0.7-1.2, p=0.6$ ) achieved independent predictor status (table 2).

\section{Discussion}

To date there are no clearly established criteria for selecting RARP over ORP. We investigated patient characteristics within the SEER-Medicare-linked database to examine potential differences between RARP and ORP patients. Our analyses detected several important findings.

First, important patient characteristics differed between RARP and ORP. Specifically, RARP patients were younger, had higher income, higher education, and were more likely to reside in urban areas than those who opted for ORP. Taken together, these observations indicate that important patient characteristics may favor access to RARP. Our data cannot determine whether such patients more aggressively seek RARP or whether they are more frequently selected for RARP by their treating surgeons.

Second, patients treated with RARP also had more favorable clinical characteristics. Specifically, RARP patients more frequently had PSA $\leq 10 \mathrm{ng} / \mathrm{ml}$ (75.0 vs. $69.3 \%$, $\mathrm{p}<0.001)$ and clinical tumor stage $\leq \mathrm{cT} 2 \mathrm{a}(88.8$ vs. $84.9 \%$, $\mathrm{p}<0.001)$ than ORP patients. This observation may indicate a selection bias toward more favorable clinical characteristics when RARP is contemplated. Alternatively, it may suggest that patients with more aggressive PCa characteristics opt for ORP or that surgeons recommend ORP to such patients more frequently.

Third, with respect to pathological and surgical characteristics, a statistically and significantly lower proportion of patients with lymph node invasion were treated with RARP ( 1.4 vs. $3.4 \%, \mathrm{p}<0.001)$. The substantially lower rate of PLND within the RARP patients (49.4 vs. $71.8 \%, \mathrm{p}<0.001)$ explains the latter observation. Although, RARP by itself should not limit PLND rates
Schiffmann et al. 
Table 1. Descriptive statistics of 5,915 patients treated with RP for localized prostate cancer performed between October 1, 2008 and December 31, 2009, within the SEER-Medicare-linked database stratified according to the surgical approach

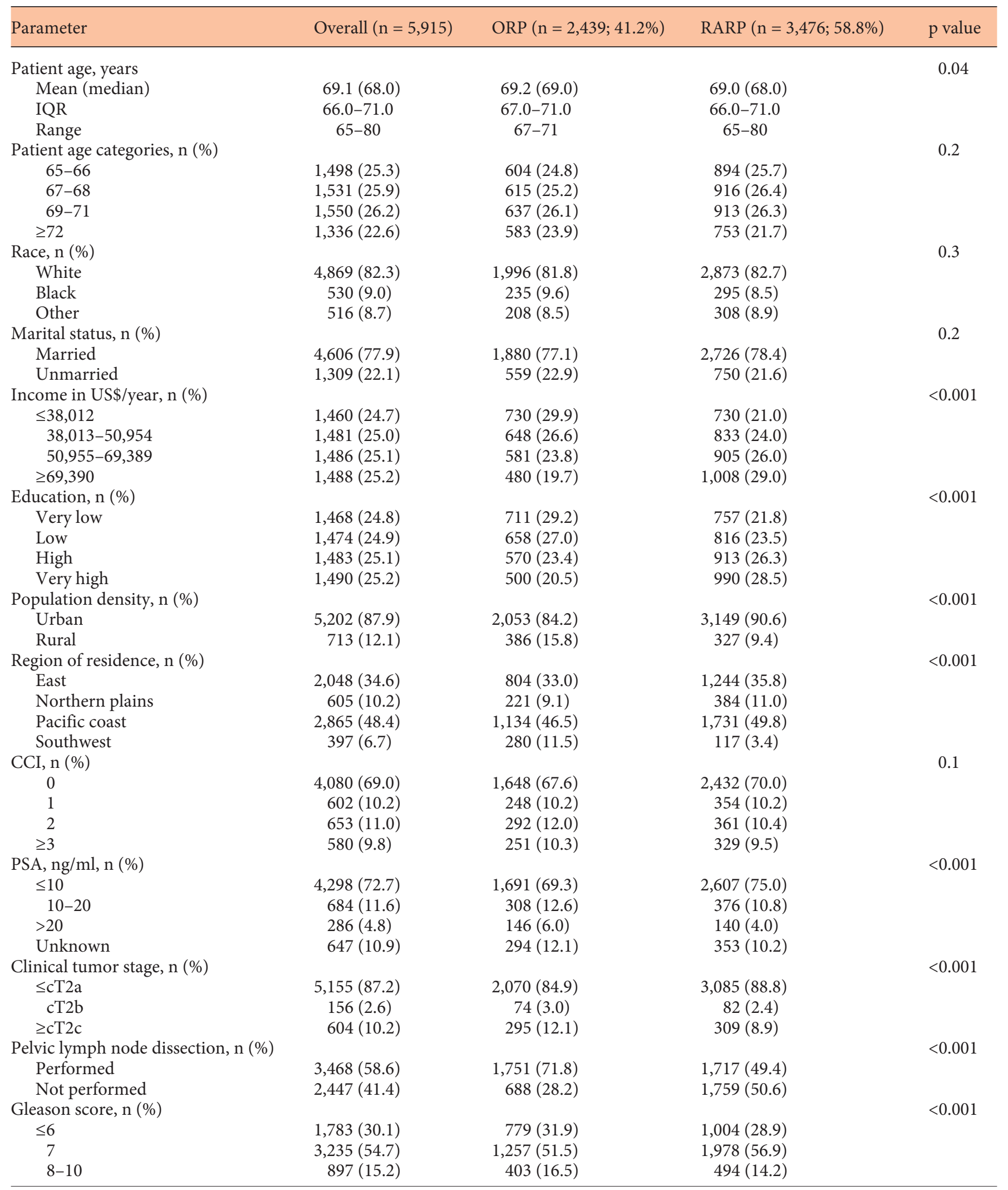


Table 1. (continued)

\begin{tabular}{|c|c|c|c|c|}
\hline Parameter & Overall $(n=5,915)$ & ORP $(n=2,439 ; 41.2 \%)$ & RARP $(\mathrm{n}=3,476 ; 58.8 \%)$ & $\mathrm{p}$ value \\
\hline Pathological tumor stage, $\mathrm{n}(\%)$ & & & & 0.006 \\
\hline $\mathrm{pT} 2$ & $4,054(68.5)$ & $1,678(68.8)$ & $2,376(68.4)$ & \\
\hline pT4 & $56(0.9)$ & $28(1.1)$ & $28(0.8)$ & \\
\hline Missing & $425(7.2)$ & $201(8.2)$ & $224(6.4)$ & \\
\hline Nodal stage, n (\%) & & & & $<0.001$ \\
\hline \multicolumn{5}{|l|}{ Organ-confined tumor } \\
\hline$(\leq \mathrm{pT} 2, \mathrm{~N} 0 / \mathrm{NX}), \mathrm{n}(\%)$ & $4,025(68.0)$ & $1,658(68.0)$ & $2,367(68.1)$ & 0.9 \\
\hline Surgical volume, $\mathrm{n}(\%)$ & & & & $<0.001$ \\
\hline Low & $875(34.1)$ & $423(37.1)$ & $452(31.7)$ & \\
\hline Intermediate & $836(32.6)$ & $468(41.1)$ & $368(25.8)$ & \\
\hline
\end{tabular}

[12-14], several authors reported lower PLND rates at RARP than ORP $[10,11,19]$. These differences may be due to a learning curve effect and may eventually disappear with increasing surgeon experience $[14,20]$. For now, if PLND is clearly indicated, ORP might represent a safer choice.

RARP patients were also more likely to harbor more favorable Gleason score ( $\leq 7)$ (85.8 vs. $83.4 \%, \mathrm{p}<0.001)$. Nonetheless, a surprisingly higher proportion of RARP patients were classified as pathological tumor stage pT3 ( 24.4 vs. $21.8 \%, \mathrm{p}=0.006$ ). This finding appears counterintuitive, unless the pathological assessment of the RARP specimens is more meticulous than that of ORP specimens.

It was not surprising to observe that a substantially and highly statistically and significantly higher proportion of RARP patients were treated by high-volume surgeons ( 42.5 vs. $21.8 \%, \mathrm{p}<0.001)$. This observation may suggest that RARP is more frequently offered by highly skilled surgeons, who perform a higher number of RARPs on an individual basis.

To the best of our knowledge, this is the first study that relied on the SEER-Medicare database that focused on explicit differences between RARP and ORP patients. However, several other authors relied on the SEER-Medicare database to examine other endpoints, such as PLND and surgical volume $[10,11,19]$. For example, $\mathrm{Hu}$ et al. [19] relied on SEER-Medicare data of 5,448 PCa patients treated between 2004 and 2006 with either ORP (72.5\%) or minimally invasive RP (MIRP) (27.5\%) without being able to truly identify RARP due to the absence of RARP specific codes. Overall, PLND was more frequently performed in patients treated with ORP compared to those treated with MIRP ( 80 vs. $43.3 \%$ ). Additionally, it was also recorded that MIRP was more often performed by surgeons from the highest surgical volume strata compared to those treated with ORP (55.0 vs. $31.5 \%$, p < 0.001). Our analysis updates these findings with special focus on RARP that is validated by the RARP-specific code. Similarly, higher PLND rates at RARP vs. ORP were also recorded in single-institutional series [21-23].

Additionally, our data validate previous observations from institutional studies. For example, Sooriakumaran et al. [6] relied on 22,393 PCa patients treated between 2000 and 2011 at 14 different centers of excellence. Of those, 7,697 (34.4\%) men were treated with RARP, 4,918 (22.0\%) with LARP and 9,778 (43.6\%) with ORP, respectively. Patients who opted for RARP were less likely to harbor high-risk PCa vs. those who opt for LARP respectively ORP (7.0 vs. 11.2 vs. $13.3 \%, \mathrm{p}<0.001)$. Unlike in our study, patients treated with RARP were less likely to harbor pT3 disease (5.0 vs. 6.9 vs. $11.1 \%, \mathrm{p}<0.001$ ). Finally, like in our study, those patients who opted for RARP were less likely to harbor Gleason score $\geq 8$ ( 7.7 vs. 6.2 vs. $10.0 \%, \mathrm{p}<0.001)$.

Regarding age, Smith et al. [24] compared 1,747 PCa patients treated at a single institution between 2002 and 2006 with either RARP $(n=1,238)$ or ORP $(n=509)$. Unlike in our study, no differences according to age were recorded between RARP and ORP patients $(p=0.3)$. Sev-
44

Urol Int 2017;98:40-48 DOI: $10.1159 / 000447495$
Schiffmann et al. 


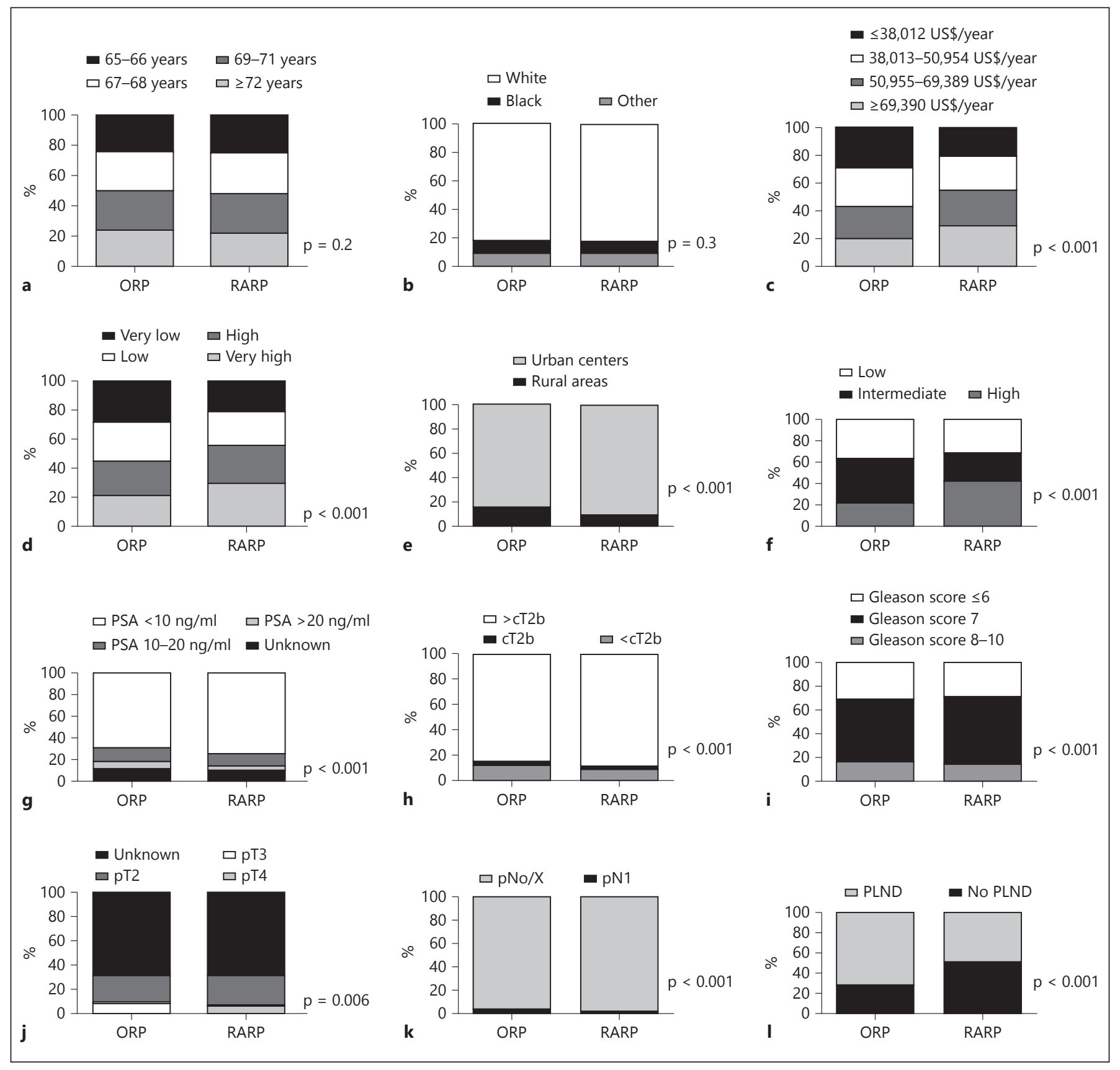

Fig. 1. Descriptive differences between 5,915 PCa patients treated with RP performed between October 1, 2008 and December 31, 2009, within the SEER-Medicare-linked database stratified according to RARP vs. ORP, according to age (a), race (b), annual income (c), education (d), population density (e), surgical volume (f), PSA level (g), clinical tumor stage (h), Gleason score (i), pathological tumor stage $(\mathbf{j})$, nodal stage $(\mathbf{k})$ and PLND (I), respectively. eral others also found no significant differences in age between RARP and ORP patients [21, 24].

Conversely, with respect to age and comorbidities, Briganti et al. [22] found RARP patients were younger and healthier. The authors relied on 2,511 PCa patients treated between 2006 and 2010 with either RARP (25.4\%) or ORP (74.6\%) at a single institution. RARP patients were younger (median 62.6 vs. 65.7 years, $\mathrm{p}<0.001$ ), healthier (CCI $\geq 2: 6.9$ vs. $10.9 \%, \mathrm{p}<0.001$ ), had lower median body mass index ( 25 vs. $26, \mathrm{p}<0.001)$ and were 
Table 2. Univariable and multivariable logistic regression analyses evaluating the predictors of RARP in 5,915 patients treated with RP for localized prostate cancer between October 1, 2008 and December 31, 2009, within the SEER-Medicare-linked database

\begin{tabular}{|c|c|c|c|c|}
\hline & \multicolumn{2}{|c|}{ Univariable analyses } & \multicolumn{2}{|c|}{ Multivariable analyses } \\
\hline & OR (95\% CI) & $\mathrm{p}$ value & OR $(95 \% \mathrm{CI})$ & $\mathrm{p}$ value \\
\hline \multicolumn{5}{|l|}{ Surgical volume } \\
\hline Low & 1 (ref.) & & 1 (ref.) & \\
\hline Intermediate & $0.7(0.6-0.9)$ & 0.002 & $0.7(0.6-0.9)$ & 0.001 \\
\hline High & $2.9(1.9-2.8)$ & $<0.001$ & $2.2(1.8-2.7)$ & $<0.001$ \\
\hline \multicolumn{5}{|l|}{ Region of residence } \\
\hline East & 1 (ref.) & & 1 (ref.) & \\
\hline Norther plain & $1.1(0.9-1.4)$ & 0.2 & $1.4(1.1-1.8)$ & 0.02 \\
\hline Pacific coast & $1.0(0.9-1.1)$ & 0.8 & $0.9(0.7-1.1)$ & 0.3 \\
\hline Southwest & $0.3(0.2-0.3)$ & 0.3 & $0.4(0.3-0.6)$ & $<0.001$ \\
\hline \multicolumn{5}{|l|}{ Education } \\
\hline Very low & 1 (ref.) & & 1 (ref.) & \\
\hline Low & $1.2(1.0-1.3)$ & 0.04 & $1.1(0.8-1.3)$ & 0.7 \\
\hline High & $1.5(1.3-1.7)$ & $<0.001$ & $1.4(1.1-1.9)$ & 0.01 \\
\hline Very high & $1.9(1.6-2.2)$ & $<0.001$ & $1.5(1.1-2.1)$ & 0.02 \\
\hline \multicolumn{5}{|l|}{ Population density } \\
\hline Urban & 1 (ref.) & & 1 (ref.) & \\
\hline Rural & $0.6(0.5-0.7)$ & $<0.001$ & $0.7(0.5-0.9)$ & 0.005 \\
\hline \multicolumn{5}{|l|}{ Clinical tumor stage } \\
\hline$\leq \mathrm{cT} 2 \mathrm{a}$ & 1 (ref.) & & 1 (ref.) & \\
\hline cT2b & $0.7(0.5-1.0)$ & 0.07 & $0.6(0.4-1.0)$ & 0.053 \\
\hline$\geq \mathrm{cT} 2 \mathrm{c}$ & $0.7(0.6-0.8)$ & $<0.001$ & $0.7(0.5-0.9)$ & 0.006 \\
\hline \multicolumn{5}{|l|}{ Patient age, years } \\
\hline $65-66$ & 1 (ref.) & & 1 (ref.) & \\
\hline $67-68$ & $1.0(0.9-1.2)$ & 0.9 & $0.9(0.7-1.1)$ & 0.3 \\
\hline $69-71$ & $1.0(0.8-1.1)$ & 0.7 & $0.8(0.7-1.1)$ & 0.1 \\
\hline$\geq 72$ & $0.9(0.8-1.0)$ & 0.07 & $0.8(0.6-1.0)$ & 0.059 \\
\hline \multicolumn{5}{|l|}{ PSA, $n g / m l$} \\
\hline$<10$ & 1 (ref.) & & 1 (ref.) & \\
\hline $10-20$ & $0.8(0.7-0.9)$ & 0.005 & $0.98(0.8-1.3)$ & 0.9 \\
\hline$>20$ & $0.6(0.5-0.8)$ & $<0.001$ & $0.8(0.6-1.2)$ & 0.3 \\
\hline Unknown & $0.8(0.7-0.9)$ & 0.003 & $0.8(0.6-1.0)$ & 0.07 \\
\hline \multicolumn{5}{|l|}{ CCI } \\
\hline 0 & 1 (ref.) & & 1 (ref.) & \\
\hline 1 & $1.0(0.8-1.2)$ & 0.7 & $0.9(0.7-1.2)$ & 0.6 \\
\hline 2 & $0.8(0.7-1.0)$ & 0.04 & $0.8(0.6-1.0)$ & 0.08 \\
\hline$\geq 3$ & $0.9(0.8-1.1)$ & 0.2 & $0.9(0.7-1.2)$ & 0.6 \\
\hline \multicolumn{5}{|l|}{ Race } \\
\hline White & 1 (ref.) & & 1 (ref.) & \\
\hline Black & $0.9(0.7-1.1)$ & 0.1 & $0.9(0.7-1.2)$ & 0.5 \\
\hline Other & $1.0(0.9-1.2)$ & 0.8 & $1.2(0.9-1.6)$ & 0.3 \\
\hline \multicolumn{5}{|l|}{ Marital status } \\
\hline Married & 1 (ref.) & & 1 (ref.) & \\
\hline Unmarried & $0.9(0.8-1.1)$ & 0.2 & $0.96(0.8-1.2)$ & 0.7 \\
\hline \multicolumn{5}{|l|}{ Income in US\$/year } \\
\hline$\leq 38,012$ & 1 (ref.) & & 1 (ref.) & \\
\hline $38,013-50,954$ & $1.3(1.1-1.5)$ & 0.001 & $0.99(0.8-1.3)$ & 0.97 \\
\hline $50,955-69,389$ & $1.6(1.4-1.8)$ & $<0.001$ & $1.3(0.95-1.7)$ & 0.1 \\
\hline$\geq 69,390$ & $2.1(1.8-2.4)$ & $<0.001$ & $1.2(0.9-1.8)$ & 0.2 \\
\hline
\end{tabular}


less likely to harbor high-risk PCa (5.8 vs. $24.1 \%$, p < 0.001) compared to those treated with ORP.

The clinical implications of our findings are manifold. First, our findings show that higher education provides better access to RARP. This finding warrants attention at the level of access to RARP. Less well-educated individuals should be given higher consideration or should be encouraged to opt for RARP, if indicated.

Second, more favorable clinical characteristics of RARP patients indicate a selection bias toward better surgical candidates. This dissemination may indicate that RARP surgeons prefer to treat patients with more favorable characteristics. Alternatively, this may indicate that RARP surgeons avoid operating patients with more complex or challenging pathology.

Third, lower rates of PLND indicate the potentially increased technical difficulty of RARP vs. ORP. The highly significant difference in PLND rates cannot be expected by more favorable clinical stage prior to RARP, alone.

Despite its strengths, our study has limitations. First, our cohort exclusively originates from the Medicare, with an age distribution of over 65 years. The results might not be generalizable to younger patients. However, PCa rates are highest in people aged $65-74$ years with a median age at diagnosis of 66 years [25]. Second, additional limitations, known to affect SEER-Medicare derived analyses are also operational, such as for example use of claims data or lack of detailed parameters that are included in prospective trials. For example, the individ- ual access to RARP treatment or distance to next RARP from the region of residence was not available within our data. Additionally, we cannot report if patients had to pay an additional fee to be treated with RARP. Third, since availability of RARP may differ substantially in other countries, our results might not be generalizable to other populations. Finally, other limitations are also operational.

\section{Conclusion}

RARP may be preferred by highly educated, urban residents from the East or Northern Plains of the United States. Those individuals may have better access to RARP. Additionally, RARP is more frequently performed in patients with more favorable PCa characteristics and RARP patients are more frequently treated by surgeons with a high volume of patients. Conversely, RARP patients less frequently benefit from PLND. These observations warrant further attention to decrease the effect of potential barriers to RARP and/or optimize RARP use.

\section{Disclosure Statement and Ethical Standards}

The authors declare that there are no financial disclosures or conflict of interest regarding this manuscript. The study was performed in accordance with the ethical standards laid down in the 1964 Declaration of Helsinki and its later amendments.

\section{References}

1 Mohler J, Bahnson RR, Boston B, et al: NCCN clinical practice guidelines in oncology: prostate cancer. J Natl Compr Canc Netw 2010;8: 162-200.

2 Heidenreich A, Bastian PJ, Bellmunt J, et al: EAU guidelines on prostate cancer. Part 1: screening, diagnosis, and local treatment with curative intent-update 2013. Eur Urol 2014; 65:124-137.

3 Lowrance WT, Eastham JA, Savage C, et al: Contemporary open and robotic radical prostatectomy practice patterns among urologists in the United States. J Urol 2012;187:20872092.

4 Trinh QD, Sammon J, Sun M, et al: Perioperative outcomes of robot-assisted radical prostatectomy compared with open radical prostatectomy: results from the nationwide inpatient sample. Eur Urol 2012;61:679685.

5 Tewari A, Sooriakumaran P, Bloch DA, et al: Positive surgical margin and perioperative complication rates of primary surgical treatments for prostate cancer: a systematic review and meta-analysis comparing retropubic, laparoscopic, and robotic prostatectomy. Eur Urol 2012;62:1-15.

6 Sooriakumaran P, Srivastava A, Shariat SF, et al: A multinational, multi-institutional study comparing positive surgical margin rates among 22393 open, laparoscopic, and robotassisted radical prostatectomy patients. Eur Urol 2014;66:450-456.

7 Bütow Z, Schunk S, Janssen M, et al: Quality of preoperative biopsy is a risk factor for positive surgical margins in organ-confined prostate cancer treated with nerve-sparing robot-assisted radical prostatectomy. Urol Int 2015;95:465-471.

8 Schiffmann J, Haese A, Leyh-Bannurah SR, et al: Adherence of the indication to European Association of Urology guideline recommended pelvic lymph node dissection at a high-volume center: differences between open and robot-assisted radical prostatectomy. Eur J Surg Oncol 2015;41:1547-1553.

9 Schiffmann J, Haese A, Lenz J, et al: Differences in patient characteristics among men choosing open or robot-assisted radical prostatectomy in contemporary practice at a European high-volume center. Urol Int 2016;97:8-15.

10 Gandaglia G, Trinh QD, Hu JC, et al: The impact of robot-assisted radical prostatectomy on the use and extent of pelvic lymph node dissection in the 'post-dissemination' period. Eur J Surg Oncol 2014;40:1080-1086.

11 Feifer AH, Elkin EB, Lowrance WT, et al Temporal trends and predictors of pelvic lymph node dissection in open or minimally invasive radical prostatectomy. Cancer 2011; 117:3933-3942.

12 Yee DS, Katz DJ, Godoy G, et al: Extended pelvic lymph node dissection in robotic-assisted radical prostatectomy: surgical technique and initial experience. Urology 2010; 75:1199-1204. 
13 Feicke A, Baumgartner M, Talimi S, et al: Robotic-assisted laparoscopic extended pelvic lymph node dissection for prostate cancer: surgical technique and experience with the first 99 cases. Eur Urol 2009;55:876-883.

14 Ploussard G, Briganti A, de la Taille A, et al: Pelvic lymph node dissection during robotassisted radical prostatectomy: efficacy, limitations, and complications-a systematic review of the literature. Eur Urol 2014;65:716.

$15 \mathrm{Hu}$ JC, Gu X, Lipsitz SR, et al: Comparative effectiveness of minimally invasive vs open radical prostatectomy. JAMA 2009;302: 1557-1564.

16 Fowler FJ Jr, Gerstein BS, Barry MJ: How patient centered are medical decisions?: results of a national survey. JAMA Intern Med 2013; 173:1215-1221.

17 Warren JL, Klabunde CN, Schrag D, et al: Overview of the SEER-Medicare data: content, research applications, and generalizability to the United States elderly population. Med Care 2002;40(8 suppl):IV-3-IV-18.
18 Klabunde CN, Potosky AL, Legler JM, et al: Development of a comorbidity index using physician claims data. J Clin Epidemiol 2000; 53:1258-1267.

$19 \mathrm{Hu}$ JC, Prasad SM, Gu X, et al: Determinants of performing radical prostatectomy pelvic lymph node dissection and the number of lymph nodes removed in elderly men. Urology 2011;77:402-406.

20 Merseburger AS, Herrmann TR, Shariat SF, et al: EAU guidelines on robotic and single-site surgery in urology. Eur Urol 2013;64:277291.

21 Williams SB, Chen MH, D'Amico AV, et al: Radical retropubic prostatectomy and robotic-assisted laparoscopic prostatectomy: likelihood of positive surgical margin(s). Urology 2010;76:1097-1101.
22 Briganti A, Bianchi M, Sun M, et al: Impact of the introduction of a robotic training programme on prostate cancer stage migration at a single tertiary referral centre. BJU Int 2013; 111:1222-1230.

23 Cooperberg MR, Kane CJ, Cowan JE, et al: Adequacy of lymphadenectomy among men undergoing robot-assisted laparoscopic radical prostatectomy. BJU Int 2010;105:88-92.

24 Smith JA Jr, Chan RC, Chang SS, et al: A comparison of the incidence and location of positive surgical margins in robotic assisted laparoscopic radical prostatectomy and open retropubic radical prostatectomy. J Urol 2007; 178:2385-2389; discussion 2389-2390.

25 Howlader NNA, Krapcho M, Garshell J, Neyman N, Altekruse SF, Kosary CL, Yu M, Ruhl J, Tatalovich Z, Cho H, Mariotto A, Lewis DR, Chen HS, Feuer EJ, Cronin KA (eds): SEER Cancer Statistics Review, 1975-2010. National Cancer Institute. http://seer.cancer.gov/ csr/1975_2010/, based on November 2012 SEER data submission, posted to the SEER web site, 2013. 